\title{
Trapianto renale da donatore vivente
}

\author{
Aris Tsalouchos ${ }^{1}$, Maurizio Salvadori² \\ ${ }^{1}$ Azienda UsI Toscana Centro, S.O.C. Nefrologia e Dialisi, Firenze II, Ospedale Santa Maria Annunziata, Bagno a Ripoli, Firenze - Italy \\ 2Professore di Nefrologia già Direttore Nefrologia e Trapianto, Azienda Ospedaliera Careggi, Firenze - Italy
}

\begin{abstract}
Kidney transplant and living donor
Kidney transplant is the best therapy to manage end-stage kidney failure. The main barriers limiting this therapy are scarcity of cadaveric donors and the comorbidities of the patients with end-stage kidney failure, which prevent the transplant. Living kidney donor transplant makes it possible to obviate the problem of scarcity of cadaveric donor organs and also presents better results than those of cadaveric transplant. The principal indication of living kidney donor transplant is preemptive transplant. This allows the patient to avoid the complications of dialysis and it has also been demonstrated that it has better results than the transplant done after dialysis has been initiated. Priority indications of living donor transplant are also twins and HLA identical siblings. We also have very favorable conditions when the donor is young and male. On the contrary, the living donor transplant will have worse results if the donors are over 60-65 years and the recipients are young, and this can be a relative contraindication. There is an absolute contraindication for the living donation when the recipient has diseases with high risk of aggressive relapse in the grafts: focal and segmental hyalinosis that had early relapse in the first transplant; atypical hemolytic uremic syndrome due to deficit or malfunction of the complement regulatory proteins; early development of glomerulonephritis due to anti-glomerular basement membrane antibody in patients with Alport syndrome; primary hyperoxaluria.

Extreme caution should also be taken in the evaluation of the kidney donors. The risks of developing renal failure or other complications are low if an adequate pre-donation evaluation has been made according to the international guidelines.
\end{abstract}

Keywords: $\mathrm{ABO}$ incompatibility, Contraindications to renal donation, Evaluation of the living donor, Living donation

\section{Introduzione}

II numero dei pazienti in attesa di trapianto di rene aumenta nel tempo e il gap fra disponibilità di organi e richiesta è una forbice che si sta allargando.

Fra le strategie per venire incontro alla domanda, da sempre c'è stato l'uso di reni da donatori viventi. Considerando gli Stati Uniti, dove l'uso di reni da donatori viventi è sempre stato relativamente diffuso, si può notare che la donazione da vivente si è ridotta del 17\% fra il 2004 e il 2014 (1). La riduzione ha riguardato soprattutto il donatore vivente consanguineo, mentre, dal 2007 in poi, è andato aumentando l'utilizzo di organi da donatore non consanguineo (2). Tuttavia,

Received: February 22, 2021

Accepted: March 2, 2021

Published online: March 30, 2021

Indirizzo per la corrispondenza:

Maurizio Salvadori

Professore di Nefrologia già Direttore Nefrologia e Trapianto

Azienda Ospedaliera Careggi

Firenze - Italy

maurizio.salvadori1@gmail.com fra il 2017 e il 2019, la donazione da vivente è nuovamente aumentata, raggiungendo i livelli più elevati (3).

Esistono, tuttavia, forti disuguaglianze nella tipologia del donatore vivente. La donazione è fatta in modo più elevato dalle donne (30 pmp), rispetto agli uomini (19 pmp) (4). II trapianto da vivente è, inoltre, meno frequente nella razza afroamericana (negli Stati Uniti), nella popolazione più giovane e nella popolazione con minori risorse economiche.

Per trovare strategie atte a ridurre tali disuguaglianze, è stata tenuta un'apposita Consensus Conference nel 2015, dalla quale sono emerse raccomandazioni atte a ridurre le disuguaglianze (5).

I riceventi di trapianto renale da donatore vivente hanno indiscutibilmente una maggiore sopravvivenza, sia dell'organo sia del ricevente, rispetto al trapianto renale da donatore cadavere. In aggiunta, il trapianto da vivente può essere eseguito molto rapidamente, evitando una lunga permanenza in dialisi. Inoltre, può essere eseguito con modalità preemptive, cioè prima dell'inizio della dialisi.

\section{Complicanze a breve termine sul donatore}

Esistono possibili, anche se infrequenti, complicanze per il donatore di rene. Possono essere complicanze a breve o 
TABELLA I - Maggiori rischi perioperatori dovuti alla nefrectomia nel donatore

\begin{tabular}{l}
\hline Emorragia \\
Ileo \\
Pneumotorace \\
Polmonite \\
Infezione del tratto urinario \\
Complicanze della ferita \\
Trombosi venosa profonda \\
Morte
\end{tabular}

a lungo termine $(6,7)$. Le complicanze a breve termine sono elencate nella Tabella I. Tali complicanze interessano il 2,5\% dei donatori e sono più frequenti nei donatori obesi, affetti da problemi della coagulazione e, in alcuni casi, con il prelievo effettuato con la nefrectomia robotica (8).

Le complicanze a lungo termine, che verranno trattate più avanti, sono state oggetto di valutazione e raccomandazioni prima da parte dell'Organ Pacific and Transplant Network (OPTN) (9) e, più recentemente, dalle KDIGO guidelines (10).

Sulla base delle KDIGO guidelines è stato possibile elaborare una valutazione del rischio per ciascun donatore, basata sul profilo demografico (età, sesso e razza) e sullo stato di salute (funzione renale, pressione arteriosa, obesità, abitudine al fumo (Fig. 1).

\section{Valutazione del donatore}

È fondamentale per la valutazione del rischio; i seguenti punti devono essere oggetto di un'accurata valutazione:
- Tipizzazione degli antigeni HLA, del gruppo sanguigno e cross-match;

- Valutazione medica, che comprenderà un'accurata anamnesi e un esame fisico, oltre a esami di laboratorio e contrastografici.

Gli esami di laboratorio dovranno essere completi e comprendere o test di tolleranza al glucosio o emoglobina glicosilata (11). Particolare attenzione sarà dedicata alle urine con attenta misurazione delle proteine urinarie. Donatori con storia di nefrolitiasi dovranno avere una valutazione dell'escrezione urinaria di calcio, ossalati, acido urico e acido citrico. Dovrà essere anche determinata la gonadotropina corionica nelle donne in premenopausa. La valutazione anatomica del rene dovrebbe includere la TC con mezzo di contrasto o la risonanza magnetica (12).

Inutile dire che dovrà essere valutata con la massima attenzione la funzione renale, anche ricorrendo a esami atti a evidenziare differenze anatomiche o di funzione dei due reni.

Attenta valutazione dovrà, poi, essere posta a evidenziare malattie infettive trasmissibili come CMV, EBV, HIV, HBV, HCV e sifilide.

Attenta valutazione dovrà poi essere posta a neoplasie preesistenti alla donazione mediante un adeguato screening. In particolare, dovrà essere posta attenzione a tumori della cervice uterina, tumori della mammella, tumori della prostata, tumori del colon e tumori del polmone.

Alcuni centri, oltre ai requisiti elencati, suggeriscono l'esecuzione di ecocardiogramma, stress da sforzo cardiaco, cistoscopia e biopsia renale in caso di dubbi sulla funzione o di malattie renali genetiche (13).

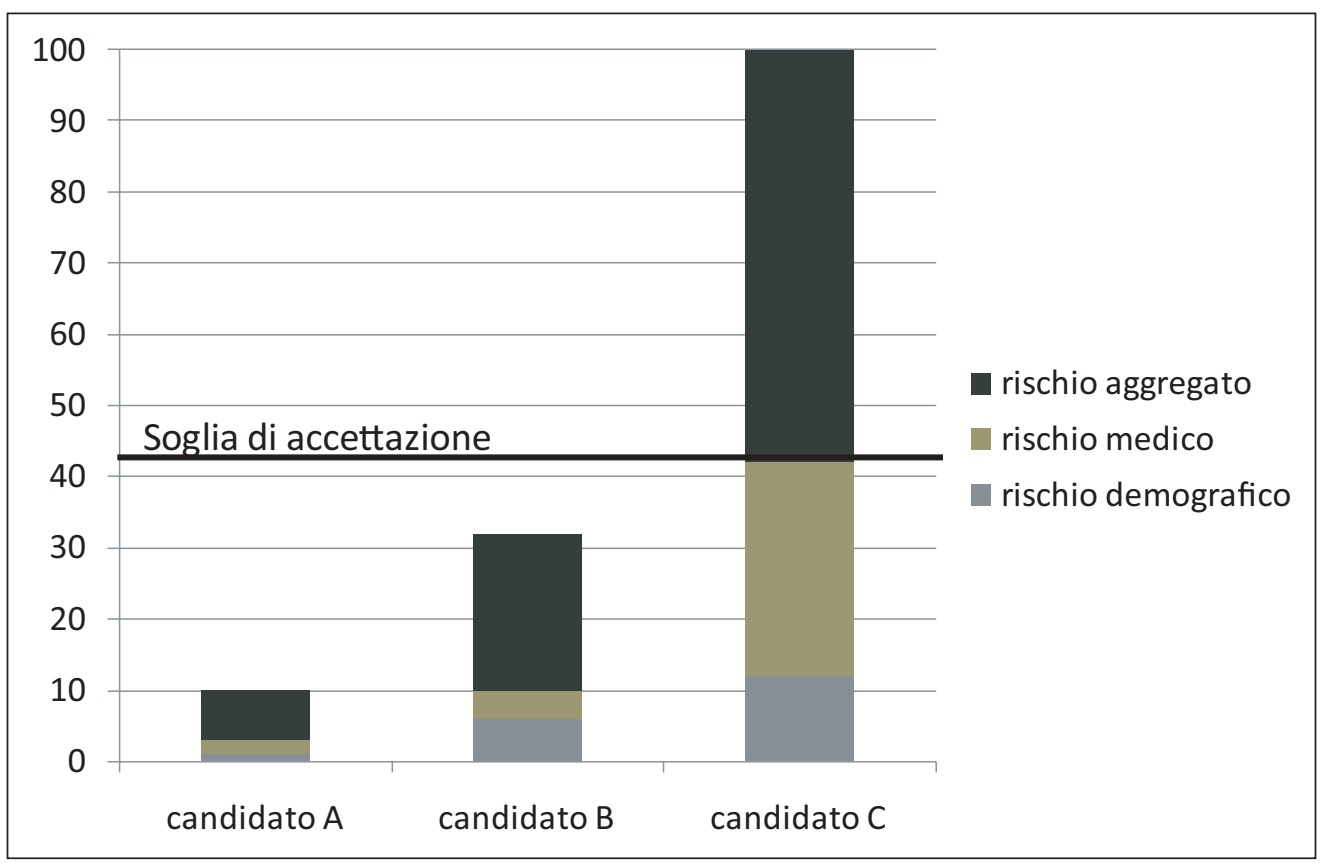

Fig. 1 - Valutazione del rischio per il donatore vivente: esemplificazione di tre candidati con valutazione dei rischi. 
- Valutazione psicosociale che dovrà comprendere:

a) valutazione dello stato mentale;

b) valutazione di comportamenti sociali a rischio per HIV, HBV e HCV (14);

c) valutazione dello stato del donatore per fumo, alcol e stupefacenti;

d) valutazione della reale e spontanea volontà di donare.

\section{Controindicazioni alla donazione}

Esistono controindicazioni assolute alla donazione di rene da vivente, come evidenziato dall'OPTN (15):

- ipertensione non controllata o storia di ipertensione con danno d'organo

- infezione da HIV

- diabete mellito

- neoplasie non completamente trattate

- infezione acuta sintomatica

- importanti anomalie psichiatriche.

Inoltre, esistono altre possibili controindicazioni o limitazioni al trapianto (16-19):

- incompatibilità $A B 0$, in assenza di un protocollo di sensibilizzazione pianificato

- proteinuria o ematuria

Donatori viventi dovrebbero avere un'escrezione di albumina urinaria inferiore ai $30 \mathrm{mg} / \mathrm{die}$. Considerando le proteine totali, una survey condotta negli Stati Uniti esclude donatori con proteine urinarie superiori ai $150 \mathrm{mg} / \mathrm{die}$ (18). L'ematuria dovrebbe essere assente. In uno studio (20), donatori viventi con più di 3 emazie per campo ad alta definizione sviluppavano, dopo la donazione, una più frequente evoluzione verso un'insufficienza renale.

Da ricordare che una microematuria persistente può celare una nefropatia glomerulare come sindrome di Alport o nefropatia da depositi di IgA. Quando sussiste il dubbio, la biopsia renale è essenziale;

- ipertensione

Ipertensione non controllata è una controindicazione al trapianto, come già detto.

L'atteggiamento nei confronti di un'ipertensione controllabile farmacologicamente è vario. Una survey eseguita negli Stati Uniti ha mostrato che più del $40 \%$ dei centri trapianto esclude pazienti che assumono farmaci per il controllo della pressione (18);

- storia di nefrolitiasi

Sempre la solita survey (18) ammette alla donazione pazienti con storia di nefrolitiasi, purché uno studio attento non documenti calcoli in atto e siano normali gli studi metabolici;

- aumento di body mass index

Pazienti con BMI >30 kg/m² dovrebbero essere esclusi, perché è stata osservata una correlazione fra obesità $\mathrm{e}$ rischio di comparsa di insufficienza renale a 20 anni dalla donazione $(21,22)$;

- neoplasie

Pazienti con neoplasie trattate, ma con rischio intermedio di trasmissione dovrebbero essere esclusi: sono pazienti con melanomi, gammopatie monoclonali e tumori del testicolo, del polmone o della mammella.

KDIGO suggerisce di ammettere caso per caso alla donazione pazienti con storia di neoplasia trattata e basso rischio di trasmissione (23).

\section{Rischio a lungo termine per il donatore}

\section{Mortalità e rischio cardiovascolare}

Gli studi effettuati suggeriscono che la sopravvivenza a lungo termine dopo donazione di rene è uguale a quella della popolazione generale (24) e questo anche per donatori di più di 55 anni, purché accuratamente selezionati (25).

Uno studio effettuato dal Chronic Renal Impairment in Birmingham (CRIB) ha osservato, nei donatori, un aumento della massa ventricolare sinistra non associato alla riduzione del filtrato glomerulare (26).

\section{Sviluppo di insufficienza renale nel donatore}

Tre studi importanti documentano che esiste, per il donatore, un rischio di sviluppare insufficienza renale.

Uno studio norvegese su 1.901 donatori di rene ha documentato un rischio di sviluppare insufficienza renale dello $0,47 \%$, confrontato con un rischio dello $0,07 \%$ nella popolazione generale (27).

Uno studio eseguito negli Stati Uniti (NHANES III) (28) ha documentato un rischio a 15 anni di 30,8/10.000 donatori, confrontato con 3,9/10.000 nella popolazione generale.

Infine, una metanalisi (24) ha documentato un rischio relativo (R.R.) per insufficienza renale di 8,83.

Su queste basi, I'OPTN raccomanda di informare accuratamente i potenziali donatori di rene.

\section{Sviluppo di ipertensione}

Gli studi su quest'aspetto non sono univoci.

Una metanalisi di 48 studi (29) non ha documentato significativi aumenti della pressione arteriosa. Al contrario, un altro studio (30) ha documentato una pressione arteriosa più elevata nei donatori che nella popolazione di controllo (16,3\% vs $11,9 \%)$.

\section{Neoplasie}

Neoplasie dopo trapianto di rene si sviluppano con minor frequenza, probabilmente per l'accurato prescreening eseguito sul donatore prima del trapianto (31). 


\section{Rischio per donatore anziano}

Uno studio effettuato su 3.368 donatori viventi di età superiore ai 55 anni non ha dimostrato a 8 anni un aumento di mortalità o di patologia cardiovascolare (25).

In uno studio americano, l'incidenza cumulativa di insufficienza renale a 15 anni per 10.000 soggetti era di 29 per donatori fra i 18 e i 39 anni, di 17 per donatori fra i 40 e i 49 anni, di 55 per donatori fra i 50 e i 59 anni e di 70 per donatori oltre i 60 anni (28).

\section{Prospettive}

Globalmente, il trapianto renale da vivente rappresenta il trattamento ottimale dell'insufficienza renale terminale e il suo impiego dovrebbe essere implementato.

Uno dei principali ostacoli al trapianto renale da vivente è che circa il $30 \%$ delle coppie è immunologicamente incompatibile o per la presenza nel ricevente di anticorpi contro il sistema HLA del donatore o per incompatibilità del gruppo sanguigno $A B O$.

Quest'ultima può essere ovviata o con adeguate tecniche di desensibilizzazione (utili anche in caso di anticorpi anti HLA) o attraverso un sistema di scambi incrociati di reni fra due o più coppie $A B 0$ incompatibili.

Quest'ultimo sistema, chiamato anche paired kidney donation, richiede un'organizzazione che non tutti i centri trapianto hanno e che spesso è organizzata su base nazionale.

La desensibilizzazione, che consiste nel rimuovere gli anticorpi presenti nel ricevente e nell'impedire che se ne formino dopo il trapianto, è il sistema più frequentemente usato. In centri adeguatamente attrezzati, può conseguire risultati simili a quelli che si hanno nei trapianti ABO compatibili (32) (Tab. II).

TABELLA II - Rischio relativo per trapianti ab0 incompatibili, confrontati con trapianti ab0 compatibili

\begin{tabular}{ll}
\hline Sopravvivenza del paziente a 1 anno & $0,99(0,98-1,00)$ \\
Sopravvivenza dell'organo a 1 anno & $0,97(0,96-0,98)$ \\
Infezioni gravi & $1,44(1,13-1,82)$ \\
Sanguinamento & $1,92(1,36-2,72)$ \\
\hline
\end{tabular}

Altri sistemi proposti consistono nel ricorrere a donatori ad alto rischio (33) o nel proporre incentivi economici (34). Entrambi i sistemi sono stati internazionalmente rifiutati per motivazioni etiche o mediche.

\section{Disclosures}

Conflict of interest: The authors declare no conflict of interest. Financial support: This research received no specific grant from any funding agency in the public, commercial, or not-for-profit sectors.

\section{Bibliografia}

1. Kasiske BL, Asrani SK, Dew MA, et al. The Living Donor Collective: A Scientific Registry for Living Donors. Am J Transplant. 2017;17(12):3040-3048. Crossref PubMed

2. Matas AJ, Smith JM, Skeans MA, et al. OPTN/SRTR 2013 Annual Data Report: kidney. Am J Transplant. 2015;15(S2)(suppl 2): 1-34. Crossref PubMed

3. Organ Procurement and Transplant Network (OPTN)/United Network for Organ Sharing (UNOS). National data reports, transplants by donor type. Online (accessed january 28, 2021).

4. Gill J, Joffres $Y$, Rose $C$, et al. The Change in Living Kidney Donation in Women and Men in the United States (20052015): A Population-Based Analysis. J Am Soc Nephrol. 2018; 29(4):1301-1308. Crossref PubMed

5. LaPointe Rudow D, Hays R, Baliga P, et al. Consensus conference on best practices in live kidney donation: recommendations to optimize education, access, and care. Am J Transplant. 2015; 15(4):914-922. Crossref PubMed

6. Lentine KL, Patel A. Risks and outcomes of living donation. Adv Chronic Kidney Dis. 2012;19(4):220-228. Crossref PubMed

7. Lentine KL, Kasiske BL, Levey AS, et al. KDIGO Clinical Practice Guideline on the Evaluation and Care of Living Kidney Donors. Transplantation. 2017;101(8S)(suppl 1):S1-S109. Crossref PubMed

8. Lentine $\mathrm{KL}$, Lam NN, Axelrod $\mathrm{D}$, et al. Perioperative Complications After Living Kidney Donation: A National Study. Am J Transplant. 2016;16(6):1848-1857. Crossref PubMed

9. Organ Procurement and Transplantation Network: Policies. Online (accessed december 27, 2020).

10. Lentine $\mathrm{KL}$, Kasiske BL, Levey AS, et al. Summary of Kidney Disease: Improving Global Outcomes (KDIGO) Clinical Practice Guideline on the Evaluation and Care of Living Kidney Donors. Transplantation. 2017;101(8):1783-1792. Crossref PubMed

11. Vigneault CB, Asch WS, Dahl NK, Bia MJ. Should living kidney donor candidates with impaired fasting glucose donate? Clin J Am Soc Nephrol. 2011;6(8):2054-2059. Crossref PubMed

12. Gluecker TM, Mayr M, Schwarz J, et al. Comparison of CT angiography with $\mathrm{MR}$ angiography in the preoperative assessment of living kidney donors. Transplantation. 2008; 86(9):1249-1256. Crossref PubMed

13. Delmonico F; Council of the Transplantation Society. A Report of the Amsterdam Forum On the Care of the Live Kidney Donor: Data and Medical Guidelines. Transplantation. 2005;79(6) (suppl):S53-S66. PubMed

14. Seem DL, Lee I, Umscheid CA, Kuehnert MJ. Excerpt from PHS guideline for reducing HIV, HBV and HCV transmission through organ transplantation. Am J Transplant. 2013;13(8):1953-1962. Crossref PubMed

15. Mağden K, Ucar FB, Velioğlu A, et al. Donor Contraindications to Living Kidney Donation: A Single-Center Experience. Transplant Proc. 2015;47(5):1299-1301. Crossref PubMed

16. Abramowicz D, Cochat P, Claas FH, et al. European Renal Best Practice Guideline on kidney donor and recipient evaluation and perioperative care. Nephrol Dial Transplant. 2015;30(11): 1790-1797. Crossref PubMed

17. van Hardeveld E, Tong A; CARI. The CARI guidelines. Psychosocial care of living kidney donors. Nephrology (Carlton). 2010;15(suppl 1):S80-S87. Crossref PubMed

18. Mandelbrot DA, Pavlakis M, Danovitch GM, et al. The medical evaluation of living kidney donors: a survey of US transplant centers. Am J Transplant. 2007;7(10):2333-2343. Crossref PubMed

19. Pascual J, Abramowicz D, Cochat P, et al. European renal best practice guideline on the management and evaluation of the 
kidney donor and recipient. Nefrologia. 2014;34(3):293-301. PubMed

20. Kido R, Shibagaki Y, Iwadoh K, et al. Persistent glomerular hematuria in living kidney donors confers a risk of progressive kidney disease in donors after heminephrectomy. Am J Transplant. 2010;10(7):1597-1604. Crossref PubMed

21. Locke JE, Reed RD, Massie A, et al. Obesity increases the risk of end-stage renal disease among living kidney donors. Kidney Int. 2017;91(3):699-703. Crossref PubMed

22. Massie $A B$, Muzaale AD, Luo X, et al. Quantifying Postdonation Risk of ESRD in Living Kidney Donors. J Am Soc Nephrol. 2017;28(9):2749-2755. Crossref PubMed

23. Nalesnik MA, Woodle ES, Dimaio JM, et al. Donor-transmitted malignancies in organ transplantation: assessment of clinical risk. Am J Transplant. 2011;11(6):1140-1147. Crossref PubMed

24. O'Keeffe LM, Ramond A, Oliver-Williams C, et al. Mid- and Long-Term Health Risks in Living Kidney Donors: A Systematic Review and Meta-analysis. Ann Intern Med. 2018;168(4):276284. Crossref PubMed

25. Reese PP, Bloom RD, Feldman $\mathrm{HI}$, et al. Mortality and cardiovascular disease among older live kidney donors. Am J Transplant. 2014;14(8):1853-1861. Crossref PubMed

26. Moody WE, Ferro CJ, Edwards NC, et al; CRIB-Donor Study Investigators. Cardiovascular Effects of Unilateral Nephrectomy in Living Kidney Donors. Hypertension. 2016;67(2):368-377. Crossref PubMed

27. Mjøen G, Hallan S, Hartmann A, et al. Long-term risks for kidney donors. Kidney Int. 2014;86(1):162-167. Crossref PubMed
28. Muzaale AD, Massie AB, Wang MC, et al. Risk of end-stage renal disease following live kidney donation. JAMA. 2014;311(6): 579-586. Crossref PubMed

29. Boudville N, Prasad GV, Knoll G, et al; Donor Nephrectomy Outcomes Research (DONOR) Network. Meta-analysis: risk for hypertension in living kidney donors. Ann Intern Med. 2006;145(3):185-196. Crossref PubMed

30. Garg AX, Prasad GV, Thiessen-Philbrook HR, et al; Donor Nephrectomy Outcomes Research (DONOR) Network. Cardiovascular disease and hypertension risk in living kidney donors: an analysis of health administrative data in Ontario, Canada. Transplantation. 2008;86(3):399-406. Crossref PubMed

31. Lentine $\mathrm{KL}$, Vijayan $\mathrm{A}$, Xiao $\mathrm{H}$, et al. Cancer diagnoses after living kidney donation: linking U.S. Registry data and administrative claims. Transplantation. 2012;94(2):139-144. Crossref PubMed

32. de Weerd AE, Betjes MGH. ABO-Incompatible Kidney Transplant Outcomes: A Meta-Analysis. Clin J Am Soc Nephrol. 2018;13(8):1234-1243. Crossref PubMed

33. Gambaro G, Zaza G, Citterio F, Naticchia A, Ferraro PM. Living kidney donation from people at risk of nephrolithiasis, with a focus on the genetic forms. Urolithiasis. 2019;47(1):115-123. Crossref PubMed

34. Przech S, Garg AX, Arnold JB, et al; Donor Nephrectomy Outcomes Research (DONOR) Network. Financial Costs Incurred by Living Kidney Donors: A Prospective Cohort Study. J Am Soc Nephrol. 2018;29(12):2847-2857. Crossref PubMed 